引用格式: 赵敏燕, 董锁成, 吴忠宏, 等. 森林体验教育活动对城市公众环境负责任行为的影响 [J]. 资源科学, 2020, 42(3): 583592. [Zhao M Y, Dong S C, Wu H C, et al. Influence of forest experience education on urban public's environmentally responsible behavior[J]. Resources Science, 2020, 42(3): 583-592.] DOI: 10.18402/resci.2020.03.16

\title{
森林体验教育活动对城市公众环境 负责任行为的影响
}

\author{
赵敏燕 ${ }^{1,2}$, 董锁成 ${ }^{1}$, 吴忠宏 ${ }^{3}$, 李 宇 ${ }^{1}$,马 红 ${ }^{4}$, 郡 丹 $^{4}$, 高 宁 $^{5}$, 夏 冰 $^{1}$
}

(1. 中国科学院地理科学与资源研究所, 北京 $100101 ; 2$. 中国科学院青藏高原研究所, 北京 $100101 ； 3$. 台中教育大学,台中 40306；4. 北京市林业碳汇工作办公室, 北京 100013 ;

5. 西南林业大学, 昆明 650224)

\begin{abstract}
摘 要: 全球可持续发展和中国生态文明教育对激发公众环境意识、引导公众环境负责任行为提出了更高要 求, 尤其对于城市公众的影响意义重大。北京市率先引入森林体验教育理念, 发挥森林生态系统文化服务功能, 开 展丰富多彩的森林体验教育活动。本文基于心流理论及其他文献, 构建了森林体验教育活动对引导公众环境负责 任行为影响的概念模型, 以北京为例调查了 676 位参与活动的公众,运用结构方程模型方法验证了参与动机 (活动 前)、活动体验 (活动中) 对环境负责任行为 (活动后) 的影响机理。研究结果表明活动体验对引导公众环境负责任 行为的影响是显著且正向的, 并且是参与动机和环境负责任行为之间关系的完全中介变量。这一结论说明了森林 体验教育活动的有效性和必要性, 为进一步提升北京市活动效果和推动中国其他地区类似活动提供了理论支撑和 实践参考。
\end{abstract}

关键词: 森林体验教育; 环境负责任行为;城市公众;心流理论;结构方程模型; 北京

DOI :10.18402/resci.2020.03.16

\section{1 引言}

全球环境事件频发致使人与自然关系日趋紧 张, 联合国 2016 年正式启动《2030 年可持续发展议 程》, 明确提出 17 项可持续发展目标, 环境保护事业 社会公众责任再度升级, 激发和引导公众环境负责 任行为 (Environmentally Responsible Behavior, ERB) 成为众多国际学者的研究热点 ${ }^{[1]}$ 。中国共谋全球生 态文明建设,强调树立尊重自然、顺应自然、保护自 然的理念, 教育引导公众环境负责任行为, 深度参 与全球环境保护事业, 促进全球可持续发展目标的 实现 ${ }^{[2]}$ 。城市公众是森林资源利用的主要群体, 也 是环境问题的承受者, 有效引导城市公众环境负责 任行为意义重大, 有助于彰显中国环境保护的使命

\section{担当,夯实中国社会公众的责任根基。}

森林体验教育理念最早由德国林业学家 Franz Heske $^{[3]}$ 在 1986 年提出, 核心理念是发挥森林生态系 统文化服务功能, 激起公众对森林的好奇心, 参与 互动有趣的体验活动, 构建走进森林、认识森林、体 验森林、保护森林的教育模式。德国经过 30 多年的 实践探索, 森林体验教育工作已成为联合国教科文 组织 “可持续发展教育” 的成功典范, 目前在日本、 韩国以及欧美多个国家被推广; 日本建立了 2000 多 所自然学校,面向各年龄阶段的青少年开展自然体 验、生态研究、灾害救援等多样的体验活动; 韩国 120 处休养林重视体验教育设施建设, 免费提供“森 林之家”、自助宿营地、休养文化会馆、露营地、探访

收稿日期: 2018-08-04, 修订日期: 2019-12-20

基金项目: 国家自然科学基金青年项目 (41801220);国家科技部科技基础资源调查专项(2017FY101303;2017FY101300)。

作者简介: 赵敏燕,女, 新疆乌鲁木齐人, 副教授, 博士后, 从事环境解说、自然教育、区域生态经济等研究。E-mail: my.z@yeah.net

通讯作者: 李宇, 男, 河北保定人, 副研究员, 博士, 硕士生导师, 从事旅游地理、区域生态经济等研究。E-mail: liy@igsnrr.ac.cn 
路等, 每年可接待 943.7 万公众 ${ }^{[4]}$ 。北京市 2010 年以 来借鉴国外经验, 引人森林体验教育概念, 各类丰 富多彩的活动涉及全市 12 个森林公园, 参与公众人 数逐年显著增长, 2018 年直接参与近 2 万人次, 间接 参与达 10 万人次, 社会公众知晓度和认可度较高 ${ }^{[5]}$ 。

纵观国内外以往研究, 游憩体验对于公众增加 自然文化知识、提升环境态度的效果方面达成了共 识, 但是帮助公众获取自然体验及引导环境负责任 行为方面的研究非常少 ${ }^{[6,7]}$ 。Juvan 等 ${ }^{[8]}$ 尝试探索有 效测量环境负责任行为的方法; Mei 等 ${ }^{[9]}$ 通过对比城 市公众和郊区公众的环境负责任行为意向, 得出影 响他们低碳行为的环境心理因素; $\mathrm{Su}$ 等 ${ }^{[10]}$ 对比第一 次到访和多次到访的公众对环境负责任行为的差 异。中国相关理论研究和实践探索尚处于起步阶 段, 国内学者更多以获取公众活动满意度高低为评 价目标, 构建公众对活动时间、地点、形式和内容等 方面评价指标体系, 运用多层次分析法或重要表现 程度分析法展开分析 ${ }^{[1,12]}$ 。但是满意度仅反映了公 众参与活动前后期望感受的对比结果, 并不能反映 公众参与活动在知识、态度和行为等方面的变化。 因此, 本文以北京市森林体验教育活动为例, 尝试 揭示森林体验教育对于城市公众行为影响的主要 因素和机理, 力图为中国森林体验教育设施和活动 设计提供科学依据和参考。

\section{2 研究综述与理论模型}

\section{1 心流理论}

美国心理学家 Csikszentmihalyi ${ }^{[13]}$ 于 1960 年代 最早提出了心流(Flow) 的概念, 即个体全身心投人 到某项活动时, 获得的一种对活动人迷、全神贯注、 注意力高度集中、活动流畅高效的深层沉浸心理体 验, 随之达到一种活动与意识融合、时间感消失和 忘我的境界, 也称为沉浸理论。简单地说, 心流是 全神贯注投人而更好完成任务的一种心理状态, 最 佳“心流”体验产生需具备 3 个条件: (1)清晰明确的 目标, (2)准确及时的反馈, (3)与技能相平衡的挑 战。“心流”体验被广泛运用到公众体验交互设计方 法中, 比如活动设计、体育运动、游戏产品等。Novak 等 ${ }^{[14]}$ 依据心流体验的过程, 提取了“心流”体验的 9 个特征, 并系统归纳为 3 类因素, 即条件因素、体验 因素和结果因素, 其中条件因素可被控制, 体验因
素可被加强, 激发心流体验的产生, 获得更佳的结 果因素(图 1)。

心流理论应用到森林体验教育活动将有助于 提高公众的认知水平、积极情绪、活动满意度,也有 助于服务供给方定位目标受众和加强体验结果。 国外运用心流理论探究 3 类因素之间的关系, 区分 公众在活动过程中的意识和行为状态, 得出优化活 动内涵和形式的综合依据, 帮助公众获取最佳心流 体验, 指导活动提升体验效果 ${ }^{[13,14]}$ 。然而国内针对 3 类因素之间关系的研究比较薄弱,尤其是针对中间 层次体验因素特征的研究较少,致使体验内涵的相 对统一性和体验类型的多样化之间常常难以有效 协调 ${ }^{[15]}$ 。

\section{2 森林体验教育活动}

森林体验教育活动是基于心流理论在森林自然 环境情景下调动公众丰富感官的体验教育活动。 根据心流理论, 森林体验教育活动是公众在 “活动 前一活动中一活动后” 不同阶段中个体态度及行为 意向变化的过程 ${ }^{[16]}$ 。活动前公众开始做准备,通过 书籍、网络等媒体收集森林体验活动的相关信息; 在森林环境参与活动时, 由于自身对目的地认知较 为缺乏和单一, 需要通过森林体验教育活动 (人员 媒介和非人员媒介) 满足其认知的目的, 丰富其游 览体验, 加深其对森林自然和历史文化资源的认 同; 活动结束后, 公众离开时的行为意向既可以体 现对某一事物的态度反馈, 又可以作为预测公众行 为最好的指标 ${ }^{[17]}$ 。人们的实际行为和行为意向受态 度和知觉行为控制的影响, 态度越积极、知觉行为 控制压力越大, 则行为意向越强。王屏等 ${ }^{\left[{ }^{[18}\right.}$ 从解说 驱动机制视角开展了中西森林旅游者生态行为研 究, 提出 “生态知识一生态体验一生态态度一生态

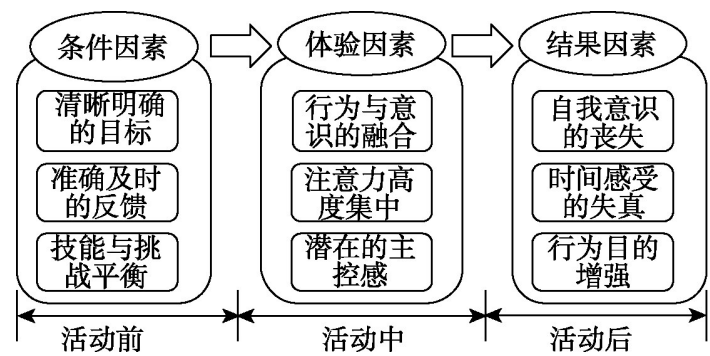

图 1 心流理论的 3 类因素

Figure 1 Three factors of the flow theory 
行为”的理论行为路径。本文基于心流理论构建概 念模型,引人森林情境变量和自我控制变量等,增 强研究多因素影响的真实环境模拟。

\section{3 环境负责任行为}

环境负责任行为是指个体或群体为推动环境 可持续发展所采取的相关行动,可通过环境教育、 个人体验与经历进行强化。国外学术界通常把环 境负责任行为称为环境行为、环境保护行为、亲环 境行为等, 虽然是不同的名称, 但内涵相近, 强调公 众通过自觉参与生态保护行动, 解决可持续发展问 题 ${ }^{[19,20]}$ 。从维度划分来看, 将环境负责任行为分为 一般环境行为 (General Behavior) 和具体环境行为 (Particular Behavior)2个方面, 学者们注重对一般环 境负责任行为的分析, 而缺乏对特定区域的具体环 境负责任行为的研究 ${ }^{[21-23]}$ 。

随着环境负责任行为及其影响因素关系的进 一步厘清,学者们研究发现公众环境负责任行为受 到参与动机、游憩体验、环境态度、地方依附感等因 素影响, 同时不同人口学特征的公众, 其环境负责 任行为意向也将有所变化 ${ }^{[24]}$ 。何学欢等 ${ }^{[2]}$ 提出了引 人中国传统文化和哲学思想到公众环境行为研究 中的必要性。Ballantyne 等 ${ }^{[17]}$ 认为公众参与动机包 括自然好奇、自然倡导和学习动机 3 个维度, 并验证 了参与动机对活动体验存在正向影响。本文提出 第 1 项假设 $(\mathrm{H} 1)$ : 参与动机正向且显著影响活动体 验。余晓婷等 ${ }^{[26]}$ 通过构建游客环境责任行为概念模 型, 得出公众参与动机对其环境负责任行为存在正 向影响的结论。学者 Falk ${ }^{[27]}$ 和 Powell 等 ${ }^{[28]}$ 是出公众 对认识欣赏自然、与自然建立联系的渴望, 对自我 实现和环境态度改变产生积极作用。本文提出第 2 项假设 $(\mathrm{H} 2)$ : 参与动机正向且显著影响环境负责任 行为。Duerden 等 ${ }^{[29]}$ 提出了直接或间接的活动体验 对公众环境行为产生具有正向加强作用。本文提 出第 3 项假设 $(\mathrm{H} 3$ 3) : 活动体验正向且显著影响环境 负责任行为。根据心流理论, 体验因素是条件因素 和效果因素的中介变量, Csikszentmihalyi 等 ${ }^{[13]}$ 认为 公众获取最佳心流体验对于调节公众动机和行为 意向具有积极作用。本文提出第 4 项假设 (H4) : 活 动体验在参与动机和环境负责任行为之间起到显 著正向中介作用。
综上,本文共提出了 4 项研究假设。但是值得 注意的是, 大多研究从西方社会情境出发,许多 ERB 的测量量表不符合中国人的思维习惯和社会 文化 (如示威游行、环保人士竞选政府领导、参加公 众聚会等),不一定适用于中国情境。如果照搬套 用西方量表, 其测量结论将失去其充分的解释力和 正确性。

\section{4 概念模型}

基于心流理论综合以往相关文献,提出森林体 验教育活动 3 类因素的 4 项假设, 即条件因素选取 参与动机作为因素变量、体验因素选取活动体验作 为因素变量、结果因素选取环境负责任行为作为因 素变量; 4 项研究假设反映条件因素、体验因素和结 果因素相互作用的关系。测量掌握公众在每个活 动阶段的意识或行为状态, 说明公众环境负责任行 为在整个活动的产生或变化响应过程, 从而建立概 念模型(图 2)。

\section{3 研究方法}

\section{1 研究地概况}

自 2010 年以来, 北京市开展了森林体验教育活 动, 由北京市林业碳汇工作办公室 (国际合作办)以 “中韩合作八达岭地区森林保护与公众教育项目” 建设为依托, 以北京公众对森林体验教育产品的需 求为出发点, 围绕森林资源多功能利用思路, 借助 国际合作交流平台,开始积极探索森林体验活动的 推广和发展途径。八达岭国家级森林公园、西山国 家级森林公园 (昌华景区)、百望山森林公园等起步 较早, 并有全国级和市级试点项目带动, 分别形成 了森林教育体验馆、自然观察径、森林“悦”读、森林 手工、“背包客”夏令营、零碳音乐季等品牌活动。

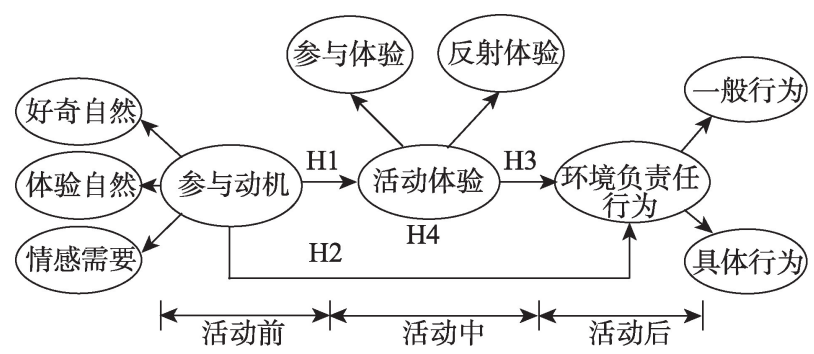

图 2 森林体验教育对城市公众环境负责任行为影响的 概念模型

Figure 2 A conceptual model of forest experience education on urban public's environmentally responsible behavior 
以北京市为研究案例地具有一定的典型性和 代表性。首先, 北京市是全国第一个引人森林体验 教育理念的地区, 自 2010 年开展各类活动, 社会公 众知晓度和认可度较高; 其次, 北京市大多数森林 公园属于典型的城镇类型, 与其他一般“自然和文 化遗产保护地体系” 的森林公园在主体功能和服务 公众的特点有所不同; 再次, 京津冀地区列入《全国 城郊森林公园发展规划(2016-2025 年)》的“优先 发展区域”。因此, 北京市森林体验教育活动以“城 市游惒型” 为主, 主要面向城市公众, 符合本文研究 需求。

\section{2 样本与数据收集}

本文采用现场纸质问卷调查法,通过测试阶段 的预调查分析、专家咨询及问卷调整, 进人正式调 查阶段, 严格控制抽样数量和质量, 保证样本代表 性和可靠性。

测试调查阶段: 于 2016 年 4 月 9 日一 24 日在北 京市西山国家级森林公园 (昌华景区、百望山景区) 的森林阅读、森林手工、森林体验活动发放测试问 卷 50 份, 回收到 39 份有效问卷, 根据测试问卷分析 结果以及与北京市园林绿化局 5 位管理人员和北京 林学会 3 位专家的访谈结果, 对测试问卷题项进行 调整和删改,形成最终的正式问卷。

正式调查阶段: 于 2016 年 4 月 30 日一-10月 23 日在北京市西山国家级森林公园 (昌华景区、百望 山景区)、八达岭国家级森林公园以及部分城市公 园共 12 个公园内开展调查 (表 1 ), 调查活动包括森 林大篷车、森林阅读、森林手工、森林大课堂、森林 体验活动等, 针对同一受众在活动前、活动中、活动 后 3 个阶段,发放 3 次不同的纸质调查问卷。

据北京市园林绿化局统计, 2015 年直接参与活
动公众共 13322 人次, 因此正式调查按照 $5 \%$ 抽样标 准计算问卷样本数量,计划回收有效问卷 666 份。 为了提高问卷代表性, 发放问卷份数提高到 1000 份,由于拒答和无效问卷等原因,仅回收问卷 760 份, 其中有效问卷 676 份,有效率 $88.9 \%$, 达到了研 究要求的样本量。

\section{3 问卷与变量测量}

测量量表是综合国内外相关研究量表, 符合中 国受众思维和作答习惯,并针对北京市森林体验教 育活动目标而逐题设计的,根据有效测试问卷分析 及专家访谈结果, 对原始维度和题项进行了初步的 删改和调整: 将“参与动机” 4 个维度删减为 3 个维 度, 删除了 “倡导自然” 维度, 相应题项进行合并; “具体行为”维度在“自备饮用水和餐食, 少用纸巾” 题项补充了“尽量少用塑料包装、纸巾和湿纸巾”, 更加符合活动实际情况, 力求达到反映北京市系列 活动特殊性和针对性的测量要求。正式问卷分为 参与动机 ( 3 个维度 8 道题项)、活动体验 ( 2 个维度 10 道题项)、环境负责任行为 ( 2 个维度 8 道题项)、 个体特征统计 (15 道题项, 获取性别、居住地、学历、 职业、月收人、总体评价等), 全问卷共 41 道题项,计 分方式均采用李克特五级量表计分法,包括“非常 符合”“符合”“一般”“不符合”和“非常不符合” 5 个 等级,保证了数据的连续性(表2)。

\section{4 结果分析}

\section{1 样本结构}

参与本次调研的 676 名公众样本的基本信息如 下: 被试对象男性占比为 $53.5 \%$, 女性占比为 $46.5 \%$; 在年龄方面, 31 45 岁以下的公众占比达 $73.5 \%$, 而 年龄在 60 岁以上和 18 岁以下的累积人数占比不足 $5.0 \%$; 在教育程度方面, 本科/专科学历的公众人数

表 1 公众有效调查问卷统计

Table 1 Statistics of the survey samples

\begin{tabular}{|c|c|c|c|}
\hline 文化活动 & 地 点 & 活动场次 & 有效问卷/份 \\
\hline 森林大篷车 & $\begin{array}{l}\text { 北京市内公园 (玉渊潭公园、玉蜓公园、望和公园、陶然亭公园、龙潭西湖公园、海淀 } \\
\text { 公园、通州大运河公园、莲花池公园、红领巾公园) }\end{array}$ & 36 & 251 \\
\hline 森林阅读 & 西山国家森林公园(百望山景区) & 4 & 110 \\
\hline 森林手工 & 西山国家森林公园(百望山景区) & 4 & 115 \\
\hline 森林大课堂 & 西山国家森林公园(百望山景区) & 3 & 124 \\
\hline \multirow[t]{2}{*}{ 森林体验活动 } & 西山国家森林公园(昌华景区)、八达岭国家森林公园 & 4 & 76 \\
\hline & 合 计 & 51 & 676 \\
\hline
\end{tabular}


表 2 公众森林体验教育活动测量量表

Table 2 Scales of the forest experience educational activities

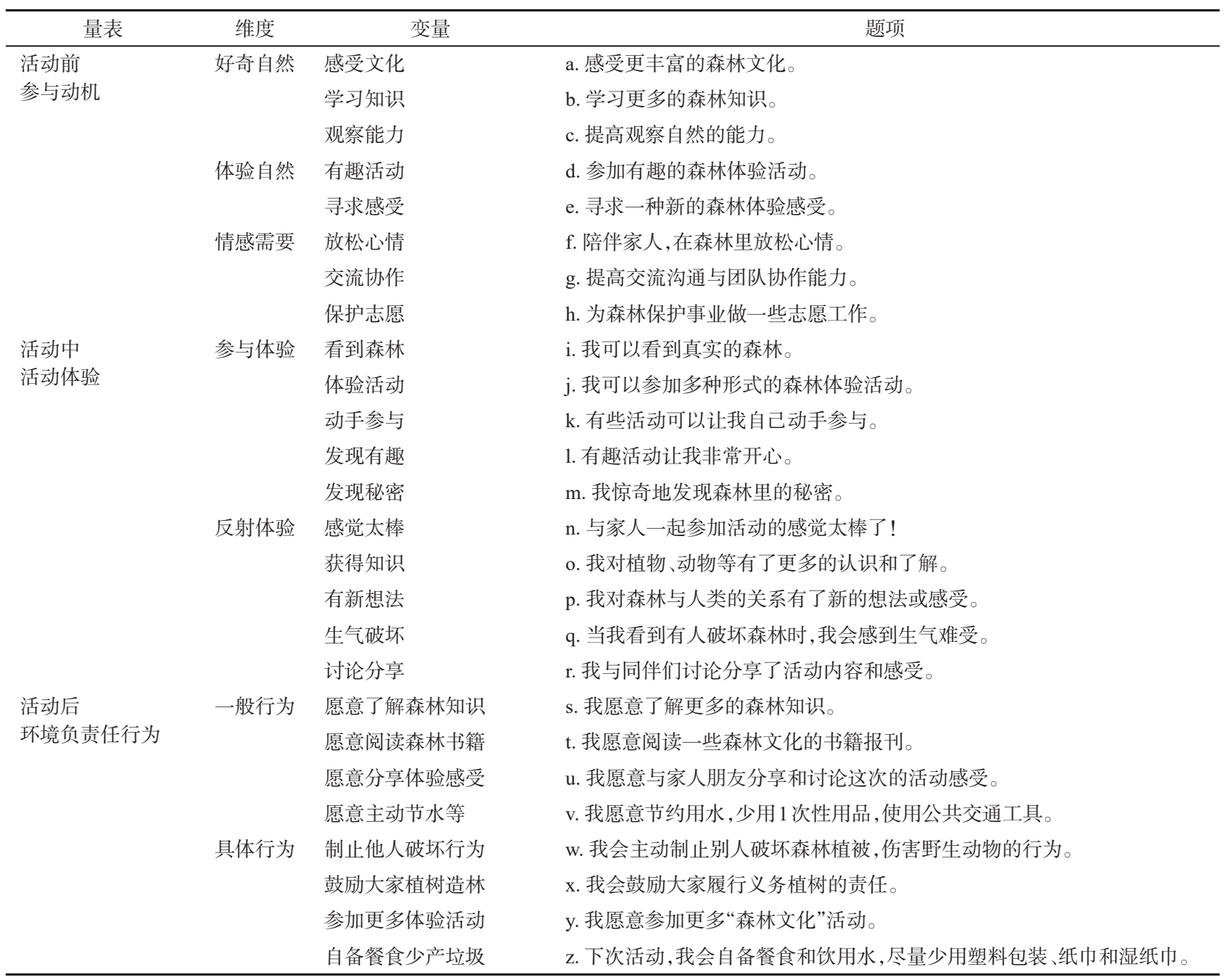

最多, 占比高达 $64.5 \%$, 超过半数游客受过高等教 育; 从职业的角度来看, 各种职业的游客占比分布 比较均匀, 游客的职业随机性较强; 从月收人水平 来看, 集中在 $0.5 \sim 1.0$ 万元之间, 占 $42.6 \%$; 从居住地 来看, $84.5 \%$ 的游客居住在北京市区, 不足 $20 \%$ 的游 客来自北京以外或者境外,样本符合城市公众的研 究需求。

\section{2 量表信度及效度检验}

\subsection{1 信度检验}

运用验证性因子分析方法对已划分好维度的 前、中、后 3 个量表进行 Cronbach’s $\alpha$ 效度检验, 结果 显示参与动机测量题项 3 个维度的 Cronbach's $\alpha$ 值 分别是 $0.940 、 0.941 、 0.941$, 活动体验测量题项 2 个 维度的 Cronbach's $\alpha$ 值分别是 $0.940 、 0.940$; 环境负 责任行为测量题项的 2 个维度的 Cronbach's $\alpha$ 分别
是 0.940 和 0.939 , 总量表的 $\alpha$ 系数为 0.942 , 全部题项 均高于 0.7 , 所有题项的相关性值都大于 0.35 , 说明 3 张量表信度一致性符合要求,具有较为理想的可靠 性和稳定性, 符合进一步检验效度的条件 ${ }^{[30,31]}$ 。

\subsection{2 效度检验}

效度检验结果看出, 3 张量表中的所有题项的 因素负荷量处在 0.5 0.95 之间,表示指标变量真实 反映其要测的维度内容。参与动机量表的 3 个维度 的组合信度值分别是 $0.862 、 0.800 、 0.720$, 平均方差 抽取量分别是 $0.676 、 0.683 、 0.502$; 活动体验量表的 2 个维度的组合信度值分别是 $0.814 、 0.859$, 平均方 差抽取量分别是 $0.501 、 0.552$; 环境负责任行为的 2 个维度的组合信度值分别是 $0.909 、 0.879$, 平均方差 抽取量分别是 $0.715 、 0.645$, 所有题项的组合信度值 全部在 0.6 以上, 平均方差抽取量值全部在 0.5 以 
上, 说明 3 张量表的内部质量优良, 效度水平达到研 究要求 $($ 表 3$)$ 。

\section{3 研究假设验证}

按照本文提出的概念模型中各个变量之间的 关系, 构建由参与动机、活动体验、环境负责任行为 构成的结构模型, 并运用 AMOS 22.0 软件对初识模 型进行运算, 在不违背经验和理论的原则下对模型 进行修正,在各维度的变量之间关联相关误差项, 严格杜绝跨维度关联误差项, 从而提升模型的拟合 度, 主要路径验证见图 3。二阶因素模型标准化路 径系数均符合良好适配标准, 结构方程模型的结果 分析看, $\chi^{2} / d f=3.480, R M R=0.031, R M S E A=0.061$, $G F I=0.901, A G F I=0.901, N F I=0.921 ， T L I=0.929$, $I F I=0.942, C F I=0.942$, 说明结构方程模型数据适配 度各项指标适配达到研究要求。
验证公众参与动机、活动体验、环境负责任行 为三者之间的影响关系 (表 4), 结果如下: (1)参与动 机对活动体验的影响在 0.05 水平下显著正向,路径 系数为 0.53 , 说明参与动机提高 1 个单位会直接激 发活动体验提升 0.53 个单位,证明 $\mathrm{H} 1$ 成立; (2)当 $P=$ 1.102 , 路径系数为 0.09 , 参与动机对环境负责任行 为的影响则不显著, 说明 $\mathrm{H} 2$ 不成立; (3)活动体验对 环境负责任行为的影响在 0.05 水平下显著正向,路 径系数为 0.67 , 说明活动体验提高 1 个单位会直接 引导环境负责任行为产生的可能性增加 0.67 个单 位,证明 $\mathrm{H} 3$ 成立。

\section{4 中介效应验证}

二阶因素模型属于完全中介效应 (Complete Mediation), 即活动体验 (活动中) 是参与动机 (活动 前)与环境负责任行为 (活动后)之间的完全中介变

表 3 量表信效度检验

Table 3 Reliability and validity test of the scales

\begin{tabular}{|c|c|c|c|c|c|}
\hline 测项 & 因素负荷量 & 信度系数 & 测量误差 & 组合信度 & 平均方差抽取量 \\
\hline 感受文化 & 0.819 & 0.671 & 0.329 & \multirow[t]{3}{*}{0.862} & \multirow[t]{3}{*}{0.676} \\
\hline 学习知识 & 0.775 & 0.601 & 0.399 & & \\
\hline 观察能力 & 0.869 & 0.755 & 0.245 & & \\
\hline 有趣活动 & 0.854 & 0.736 & 0.264 & \multirow[t]{2}{*}{0.800} & \multirow[t]{2}{*}{0.683} \\
\hline 寻求感受 & 0.795 & 0.630 & 0.370 & & \\
\hline 放松心情 & 0.724 & 0.530 & 0.470 & \multirow[t]{3}{*}{0.720} & \multirow[t]{3}{*}{0.502} \\
\hline 交流协作 & 0.647 & 0.419 & 0.581 & & \\
\hline 保护志愿 & 0.665 & 0.437 & 0.563 & & \\
\hline 看到森林 & 0.557 & 0.299 & 0.701 & \multirow[t]{5}{*}{0.814} & \multirow[t]{5}{*}{0.501} \\
\hline 体验活动 & 0.716 & 0.513 & 0.487 & & \\
\hline 动手参与 & 0.651 & 0.404 & 0.596 & & \\
\hline 发现有趣 & 0.725 & 0.511 & 0.489 & & \\
\hline 发现秘密 & 0.780 & 0.624 & 0.376 & & \\
\hline 感觉太棒 & 0.693 & 0.566 & 0.534 & \multirow[t]{5}{*}{0.859} & \multirow[t]{5}{*}{0.552} \\
\hline 获得知识 & 0.774 & 0.646 & 0.354 & & \\
\hline 有新想法 & 0.801 & 0.669 & 0.331 & & \\
\hline 生气破坏 & 0.681 & 0.436 & 0.564 & & \\
\hline 讨论分享 & 0.735 & 0.540 & 0.460 & & \\
\hline 愿意了解森林知识 & 0.864 & 0.729 & 0.271 & \multirow[t]{4}{*}{0.909} & \multirow[t]{4}{*}{0.715} \\
\hline 愿意阅读森林书籍 & 0.814 & 0.664 & 0.336 & & \\
\hline 愿意分享体验感受 & 0.824 & 0.663 & 0.337 & & \\
\hline 主动节水等行为 & 0.854 & 0.803 & 0.197 & & \\
\hline 制止他人破坏行为 & 0.824 & 0.687 & 0.313 & \multirow[t]{4}{*}{0.879} & \multirow[t]{4}{*}{0.645} \\
\hline 鼓励大家植树造林 & 0.814 & 0.663 & 0.337 & & \\
\hline 参加更多体验活动 & 0.817 & 0.651 & 0.349 & & \\
\hline 自备餐食少产垃圾 & 0.761 & 0.579 & 0.421 & & \\
\hline
\end{tabular}




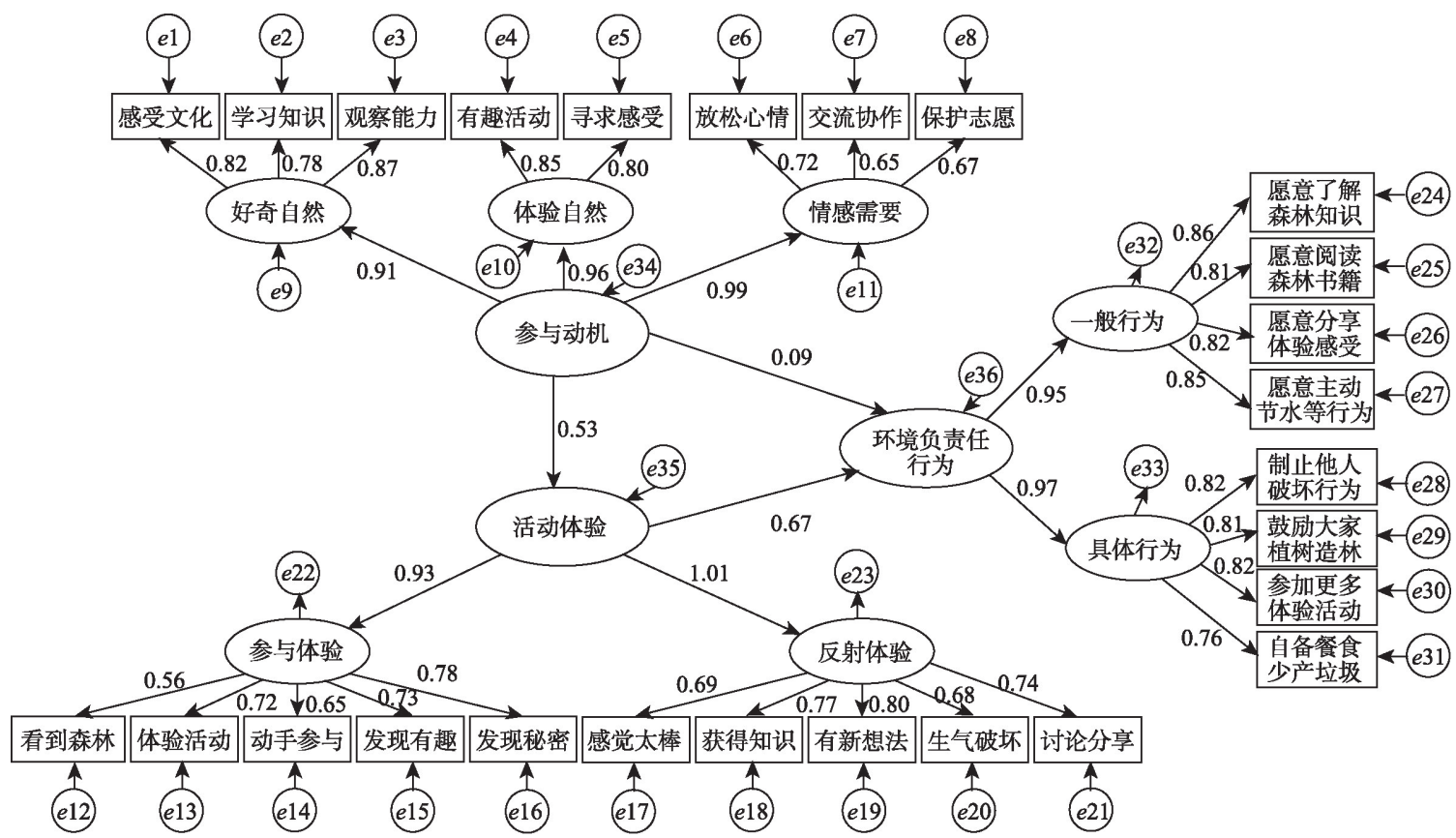

图 3 二阶因素模型标准化路径

Figure 3 Standardized paths for model of second-order factors

表 4 二阶因素模型

Table 4 Model of second-order factors

\begin{tabular}{|c|c|c|c|c|}
\hline 变量之间影响关系 & 估计值 & \multicolumn{3}{|c|}{ 标准误 临界比值显著性 } \\
\hline 活动体验〔参与动机 & 0.53 & 0.049 & 9.632 & $* * *$ \\
\hline 环境负责任行为〔参与动机 & 0.09 & 0.039 & 1.304 & 1.102 \\
\hline 环境负责任行为〔活动体验 & 0.67 & 0.067 & 11.053 & $* * *$ \\
\hline
\end{tabular}

量, 证实森林体验教育活动对于丰富公众体验及激 发环境负责任行为方面发挥了重要作用。运用 AMOS 逐步回归分析法来验证中介效应验证问 题。AMOS 运算结果显示: $P<0.05$, 路径系数为 $0.53 \times 0.67=0.355$, 故 $\mathrm{H} 4$ 成立, 即活动体验 (活动中) 是参与动机 (活动前) 与环境负责任行为 (活动后) 之间的完全中介变量, 完全中介效应路径为参与动 机 $\rightarrow$ 活动体验 $\rightarrow$ 环境负责任行为, 说明森林体验教 育活动在引导公众积极参与、激发环境负责任行为 的有效性和必要性。

因此,本文提出的 4 个理论假设中, $\mathrm{H} 1 、 \mathrm{H} 3 、 \mathrm{H} 4$ 通过假设验证, H2 没有通过假设验证。

\section{5 结论与讨论}

\section{1 结论}

本文针对北京城市公众在森林体验活动前、 中、后 3 个阶段的认知和感受,探讨森林体验教育活 动对引导公众环境负责任行为的影响机理, 得出以
下研究结论:

（1）基于心流理论,构建了森林体验教育活动 对于城市公众环境负责任行为影响的概念模型, 设 计了符合中国城市公众特色的森林体验教育活动 测试量表。用验证性因子分析法对公众活动前参 与动机、活动中活动体验、活动后环境负责任行为 的 3 个量表进行检验, 验证了各变量的测量题项均 满足效度和信度研究要求, 提高了获取数据的准确 性和可靠性。总结归纳出参与动机包括好奇自然、 体验自然和情感需要 3 个维度, 活动体验包括参与 性体验和反射性体验 2 个维度, 环境负责任行为包 括一般行为和具体行为 2 个维度。

(2)运用结构方程模型方法验证了公众参与动 机、活动体验、环境负责任行为之间的相互影响关 系。结果表明: 城市公众参与动机对环境负责任行 为的影响不显著,但对增强活动体验有贡献, 同时 说明了活动体验对城市公众环境负责任行为的影 响是显著且正向的。公众参与动机每提高 1 个单位 会直接激发活动体验提升 0.53 个单位, 活动体验每 提高 1 个单位会直接引导环境负责任行为产生的可 能性增加 0.67 个单位。

(3)证实了森林体验教育活动在引导城市公众 环境负责任行为的有效性和必要性,因为活动体验 
是公众参与动机和环境负责任行为之间关系的完 全中介变量。公众参与动机提高 1 个单位会直接引 导环境负责任行为产生的可能性增加 0.355 个单 位, 尤其是提升公众参与性体验和反射性体验, 促 进引导环境负责任行为产生的可能性, 活动后的跟 踪学习不容忽视。

\section{2 讨论}

北京市森林体验教育活动, 其活动区域属于典 型的城镇类型, 活动服务对象是以城市公众为主要 目标群体, 符合《北京城市总体规划 (2016-2035 年)》实现城市可持续发展、建设国际一流的和谐宜 居之都的发展定位。

基于本文结论,将理论研究成果转化成为实践 依据, 探讨北京市森林体验教育活动相关工作的发 展方向:

(1) 激发参与动机, 加强宣传力度。根据北京 市森林区位优势、自然和文化资源特色, 结合公众 好奇自然(学习森林生态、感受森林文化等)、体验 自然 (提升观察能力、参加有趣活动等)、情感需要 (放松心情、交流分享、保护意愿等) 等参与动机, 通 过微信、微博、电视、广播、网站等多种渠道, 加强森 林体验教育活动的宣传力度, 提供活动内容、参与 方式等便利信息, 调动公众对森林体验教育活动的 参与动机和兴趣, 产生走进森林、体验森林的内心 期望 ${ }^{[32]}$ 。

(2) 提升活动体验, 调动五感设计。根据研究 结果, 全方位提升森林体验教育活动体验, 让公众 调动五感 (听、说、读、写、触) 来感受森林的神奇, 丰 富参与性体验 (深人森林、参与不同活动等) 和反射 性体验 (产生新想法、获得新知识, 探索新秘密等) 的机会, 细致化设计体验设施和活动环节的五感体 验, 设施建设包括森林体验中心、森林课堂设施、自 然解说步道、解说牌示等硬件设施, 活动提升包括 加强自然解说员培训、开发本土森林课堂教材等; 系统化地培训活动组织人员的技能, 开发具有地方 特色的活动课程方案, 制订相关活动策划的技术规 程 ${ }^{[3,34]}$ 。

(3)引导环境行为, 形成保护氛围。此结论与 Ballantyne 等 ${ }^{[17]}$ 研究野生动物游憩体验对增加公众 短期和长期学习效果有明显作用的结论相同。活 动后跟踪公众环境负责任行为的意愿, 有益引导公
众将活动感受带到平日生活和工作之中。为他们 提供了解更多森林知识、阅读森林文化书籍报刊的 参考信息, 鼓励与家人朋友分享活动感受, 并及时 制止森林环境破坏行为。公众通过回忆、书写、分 享等过程可进行新一轮的活动体验, 加深环保知识 学习的长期效果, 游后回忆叙述有益于体验改变 ${ }^{[14,35]}$ 。 广义的森林体验教育活动并不是一时的,或者一个 闭合概念, 而是处于持续进行状态, 构建公众体验 机会谱系作为先导性工作至关重要 ${ }^{[36]}$ 。

本文完成了北京市森林体验教育活动 1 年期的 公众抽样,可在此基础上继续对北京市其他年份或 国内其他省份类似活动进行深人调查和对比研究, 为森林资源可持续利用提供科学依据。此外,城镇 森林资源、公众活动需求及特色与荒野型自然保护 地在主体功能和服务公众的特点有所不同 ${ }^{[37}$ 。目 前, 中国正处于生态文明制度改革关键时期,着力 建立以国家公园为主体的自然保护地体系,因此研 究可扩展到不同自然保护地特色情景下,提出有针 对性的公众环境负责任行为的管理模式,从而推进 国家治理体系和治理能力现代化。

\section{参考文献(References):}

[1] Han J, Lee M, Hwang Y. Tourists' environmentally responsible behavior in response to climate change and tourist experiences in nature-based tourism[J]. Sustainability, 2016, 8(7): 644-658.

[2] 于向东. 共谋全球生态文明建设的中国担当[N]. 人民日报, 2018-09-10(07). [Yu X D. China Undertakes to Build a Global Ecological Civilization[N]. People's Daily, 2018-09-10(07).]

[3] 汪清锐. 德国森林体验教育综述[J]. 林业科技情报, 2018, 50(2): 10-13. [Wang Q R. A summary of forest experience education in Germany[J]. Forestry Science and Technology Information, 2018, 50(2): 10-13.]

[4] 邹大林. 森林体验教育亟待重视: 韩国自然休养林发展的经验 与启示[J]. 绿化与生活, 2011, (11): 6-9. [Zou D L. Forest experience education needs attention: Experience and enlightenment of natural recuperation forest development in South Korea[J]. Greening and Life, 2011, (11): 6-9.]

[5] 赵悦茹, 赵敏燕, 马红, 等. 北京市森林体验教育活动分众意向 及优化模式 [J]. 环境与可持续发展, 2019, 44(1): 121- 127 . [Zhao Y R, Zhao M Y, Ma H, et al. Beijing forest experience education activities demassification perception and optimization mode [J]. Environment and Sustainable Development, 2019, 44(1): 121127.] 
[6] Kila N, Holland S M, Stein T V. Structural relationships between environmental attitudes, recreation motivations, and environmentally responsible behaviors[J]. Journal of Outdoor Recreational and Tourism, 2014, 7-8: 16-25.

[7] Lee T H, Jan F H, Huang G W. The influence of recreation experiences on environmentally responsible behavior: The case of Liuqiu Island, Taiwan[J]. Journal of Sustainable Tourism, 2015, 23(6): 947-967.

[8] Juvan E, Dolnicar S. Measuring environmentally sustainable tourist behaviour[J]. Annals of Tourism Research, 2016, 59(3): 30-44.

[9] Mei N S, Wai C W, Ahamad R B. Differential environmental psychological factors in determining low carbon behaviour among urban and suburban residents through responsible environmental behaviour model[J]. Sustainable Cities and Society, 2017, 31(3): $225-233$

[10] Su L, Swanson S R. The effect of destination social responsibility on tourist environmentally responsible behavior: Compared analysis of first- time and repeat tourists[J]. Tourism Management, 2017, 60(12): 308-321.

[11] Zhao M Y, Dong S C, Wu H C, et al. Key impact factors of visitors' environmentally responsible behaviour: Personality traits or interpretive services? A case study of Beijing's Yuyuantan Urban Park, China[J]. Asia Pacific Journal of Tourism Research, 2018, 23 (8): 792-805.

[12] Oviedo-Garcia M A, Castellanos-Verdugo M, Vega-Vazquez M, et al. The mediating roles of the overall perceived value of the ecotourism site and attitudes towards ecotourism in sustainability through the key relationship ecotourism knowledge-ecotourist satisfaction[J]. International Journal of Tourism Research, 2017, 19 (2): $203-213$.

[13] Csikzentmihalyi M. Flow: The Psychology of Optimal Experience [M]. New York: HarperCollins, 1990.

[14] Novak T P, Hoffman D L, Yung Y F. Measuring the customer experience in online environments: A structural modeling approach[J]. Marketing Science, 2000, 19(1): 22-42.

[15] 訤友猛, 谢彦君. “体验”的内涵与旅游体验属性新探[J]. 旅游学 刊, 2017, 32(11): 16-25. [Fan Y M, Xie Y J. An exploration of experience connotations and tourist experience characteristics[J]. Tourism Tribune, 2017, 32(11): 16-25.]

[16] Fishbein M, Ajzen I. Belief, Attitude, Intention, and Behavior: An Introduction to Theory and Research[M]. Reading, MA: AddisonWesley Publishing Company, 1975.

[17] Ballantyne R, Packer J, Sutherland L A. Visitors' memories of wildlife tourism: Implications for the design of powerful interpretive experiences[J]. Tourism Management, 2011, 32(4): 770-779.

[18] 王屏, 栗丽, 郭晓敏. 基于中西比较的森林公园旅游解说系统文 本研究[J]. 中国园林, 2016, 32(9): 103-107. [Wang P, Li L, Guo $\mathrm{X}$ M. Research on tourism interpretation text of forest park be- tween China and the West[J]. Chinese Landscape Architecture, 2016, 32(9): 103-107.]

[19] Weiler B, Smith L. Does more interpretation lead to greater outcomes? An assessment of the impacts of multiple layers of interpretation in a zoo context[J]. Journal of Sustainable Tourism, 2009, 17(1): 91-105.

[20] Vaske J J, Kobrin K C. Place attachment and environmentally responsible behavior[J]. Journal of Environmental Education, 2001, 32(4): 16-21.

[21] Halpenny E A. Pro-environmental behaviours and park visitors: The effect of place attachment[J]. Journal of Environmental Psychology, 2010, 30(4): 409-421.

[22] Lee T H. How recreation involvement, place attachment, and conservation commitment affect environmentally responsible behavior [J]. Journal of Sustainable Tourism, 2011, 19(7): 895-915.

[23] Ramkissoon H, Weiler B, Smith L D G. Place attachment and proenvironmental behaviour in national parks: The development of a conceptual framework[J]. Journal of Sustainable Tourism, 2012, 20 (2): 257-276.

[24] Chiu Y T H, Lee W I, Chen T H. Environmentally responsible behavior in ecotourism: Antecedents and implications[J]. Tourism Management, 2014, 40(1): 321-329.

[25] 何学欢, 胡东滨, 粟路军. 境外旅游者环境责任行为研究进展及 启示[J]. 旅游学刊, 2017, 32(9): 57-69. [He X H, Hu D B, Su L J. Research progress and the enlightenment of tourist environmentally responsible behavior (TERB) based on outbound literature[J]. Tourism Tribune, 2017, 32(9): 57-69.]

[26] 余晓婷, 吴小根, 张玉玲, 等. 游客环境责任行为驱动因素研究: 以台湾为例 [J]. 旅游学刊, 2015, 30(7): 49-59. [Yu X T, Wu X G, Zhang Y L, et al. Factors driving environmentally responsible behaviors by tourists: A case study of Taiwan, China[J]. Tourism Tribune, 2015, 30(7): 49-59.]

[27] Falk J H. Identity and the Museum Visitor Experience[M]. Walnut Creek: Left Coast Press, 2009.

[28] Powell R B, Ham S H. Can ecotourism interpretation really lead to pro- conservation knowledge, attitudes and behavior? Evidence from the Galapagos islands[J]. Journal of Sustainable Tourism, 2008, 16(4): 467-489.

[29] Duerden M D, Witt P A. The impact of direct and indirect experiences on the development of environmental knowledge, attitudes, and behavior[J]. Journal of Environmental Psychology, 2010, 30 (4): 379-392.

[30] Hair J F, Anderson R E, Tatham R L, et al. Multivariate Data Analysis[M]. London: Prentice Hall, 1998.

[31] 吴明隆. 结构方程模型: Amos 实务进阶[M]. 重庆: 重庆大学出 版社, 2013. [Wu M L. Model of Structural Equation: Amos Practice Advanced[M]. Chongqing: Chongqing University Press, 2013.]

[32] 葛学峰. 旅游目的地选择意向影响因素研究 $[D]$. 大连: 大连理 
工大学, 2012. [Ge X F. The Research of Influential Factors of Tourists Destination Choice Intention[D]. Dalian: Dalian University of Technology, 2012.]

[33] Zhao M Y, Harshaw H W, Dong W Y, et al. Visitors' satisfaction with interpretive services at the southern Sichuan bamboo sea, China[J]. Journal of Interpretation Research, 2015, 20(1): 59-65.

[34] Zhao M Y, Harshaw H W, Li Y, et al. Interpretive Satisfaction of Chinese Visitors at the Southern Sichuan Bamboo Sea: Importance-Performance Analysis Approach[C]. Kunming: Proceedings of the 2016 13th International Conference on Service Systems and
Service Management, 2016.

[35] Selstad L. The social anthropology of the tourist experience. Exploring the "middle role" [J]. Scandinavian Journal of Hospitality and Tourism, 2007, 7(1): 19-33.

[36] Lee T H, Jan F H. The low-carbon tourism experience: A multidimensional scale development[J]. Journal of Hospitality \& Tourism Research, 2019, 43(6): 890-918.

[37] Bushell R, Bricker K. Tourism in protected areas: Developing meaningful standards[J]. Tourism and Hospitality Research, 2017, 17(1): 106-120.

\title{
Influence of forest experience education on urban public's environmentally responsible behavior
}

\author{
ZHAO Minyan ${ }^{1,2}$, DONG Suocheng ${ }^{1}$, WU Homer C. ${ }^{3}$, LI Yu', MA Hong ${ }^{4}$, \\ SHAO Dan ${ }^{4}$, GAO Ning ${ }^{5}$, XIA Bing ${ }^{1}$ \\ (1. Institute of Geographic Sciences and Natural Resources Research, CAS, Beijing 100101, China; 2. Institute of Tibetan Plateau \\ Research, CAS, Beijing 100101, China; 3. Taichung University of Education, Taichung 40306, China; 4. Beijing Forestry \\ Carbon Sequestration Office, Beijing 100013, China; 5. Southwest Forestry University, Kunming 650224, China)
}

\begin{abstract}
Global sustainable development and China's ecological civilization education put forward higher requirements for stimulating public environmental awareness and guiding public's environmentally responsible behavior, especially for the urban population. Beijing took the lead in introducing the concept of forest experience education, giving full play to the forest ecosystem's educational function and carrying out rich and diversified forest experience educational activities. Based on the flow theory and literature, this study constructed a conceptual model of the impact of forest experience educational activities on guiding the environmentally responsible behavior of the public. Taking Beijing as an example, it surveyed 676 participants and used the structural equation modeling method to verify the influencing mechanism of participation motivation (before the activities) and personal experience (during the activities) on the environmentally responsible behaviors (after the activities). The results of the study indicate that the impact of experiences during such activities on guiding the public's environmentally responsible behavior is significant and positive, and it is a complete mediator between participation motivation and environmentally responsible behavior. This conclusion indicates the effectiveness and necessity of forest experience educational activities. It provides some theoretical support and practical reference for further enhancing the effect of those activities in Beijing and promoting similar activities in other areas of China.
\end{abstract}

Key words: forest experience education; environmentally responsible behavior; urban public; flow theory; structural equation model; Beijing 